ISSN 0103-9954

\title{
A IMPORTÂNCIA RELATIVA DA DENSIDADE DA MADEIRA E DO TEOR DE CARBOIDRATOS NO RENDIMENTO DE POLPA E NA QUALIDADE DO PRODUTO
}

\author{
RELATIVE IMPORTANCE OF WOOD DENSITY AND CARBOHYDRATE CONTENT ON PULPING \\ YIELD AND PRODUCT QUALITY
}

\section{Alfredo Mokfienski $^{1} \quad$ Jorge Luiz Colodette ${ }^{2}$ José Lívio Gomide ${ }^{2}$ Ana Márcia M. Ladeira Carvalho ${ }^{3}$ \\ RESUMO}

O objetivo deste estudo foi avaliar a densidade básica, a composição química e as dimensões de fibras de dez amostras de madeira de Eucalyptus spp. e, verificar o impacto desse conjunto de parâmetros e sua combinação no rendimento do processo de polpação kraft e na qualidade da polpa branqueada. A madeira de dez espécies de eucalipto, com densidade básica variando entre 365 a $544 \mathrm{~kg} / \mathrm{m}^{3}$ e teores de carboidratos totais variando entre 70,0 a 74,5\% foi transformada em polpa Kraft, com número kappa 15,516,7 e branqueada a 90\% ISO por uma seqüência de quatro estágios (OD(PO)D) composta de deslignificação com oxigênio (O), deslignificação com dióxido de cloro (D), extração alcalina com peróxido pressurizado (PO) e branqueamento com dióxido de cloro (D). A densidade básica da madeira mostrou melhor correlação com as dimensões da fibra e qualidade da polpa que a sua composição química. Porém o rendimento depurado foi mais influenciado pela composição química da madeira do que pela densidade básica. Madeiras de menor densidade apresentaram maiores rendimentos de polpação, mas o consumo específico de madeira foi mais baixo para as madeiras mais densas. Os resultados de qualidade de polpa sugerem que madeiras com densidade mais baixa devam ser direcionadas à fabricação de papel refinado (impressão e de escrita), enquanto que as mais densas sejam destinadas ao segmento de papéis sanitários (tissue).

Palavras-chave: madeira; densidade; composição química; polpa kraft.

\section{ABSTRACT}

The purpose of this study was to evaluate basic density, chemical composition and fiber dimensions of ten Eucalyptus $s p$ wood samples and to verify the impact of this set of parameters and their combination on the Kraft pulping process yield and on bleached pulp quality. Ten eucalypt woods of different species, with basic densities varying from 365 to $544 \mathrm{~kg} / \mathrm{m}^{3}$ and total wood carbohydrate contents varying from 70.0 to $74.5 \%$, were transformed into kraft pulp of kappa number 15,5-16,7 and bleached to $90 \%$ ISO brightness by the sequence $\mathrm{OD}(\mathrm{PO}) \mathrm{D}$. Wood basic density showed stronger correlations with fiber dimensions and pulp quality than did chemical composition. However, pulping yield was strongly affected by chemical composition. Lighter woods resulted in higher pulping yields. However, wood specific consumption was lower for denser woods, even though these demanded more drastic pulping conditions to achieve a given kappa number. The pulp quality results suggest that lower density woods should be directed towards fabrication of refined paper (printing and writing grades) while the denser woods should be directed to the sanitary papers segment (tissue grades).

Keywords: wood; density; chemical composition; kraft pulp.

\section{INTRODUÇÃO}

A qualidade da polpa depende da qualidade da madeira e das condições do processo de polpação. A qualidade da madeira é o resultado de práticas de manejo florestal e de uma combinação de características genéticas, físicas, anatômicas e químicas da madeira (SHIMOYAMA e BARRICHELO, 1991). As polpas de eucalipto são desejadas para fabricação de papéis para impressão e escrita e para papéis sanitários, em razão das suas características anatômicas e químicas. Tem sido mostrado que a densidade da madeira exerce uma

1. Engenheiro Florestal, Dr., Professor do Curso de Pós Graduação em Celulose e Papel, Universidade Federal de Viçosa, Caixa Postal 2037, Coqueiral, CEP 29195-000, Aracruz (ES). al.mokfi@terra.com.br

2. Engenheiro Florestal, Dr., Professor Titular do Departamento de Engenharia Florestal, Universidade Federal de Viçosa, Campus Universitário, CEP 36570, Viçosa (MG). colodett@ufv.br

3. Engenheira Florestal, Dr ${ }^{\mathrm{a}}$, Professora Adjunta do Departamento de Engenharia Florestal, Universidade Federal dos Vales do Jequitinhonha e Mucuri, Campus II - Rodovia MGT 367, Km 583, n. 5000, Bairro Alto da Jacuba, CEP 39100-000, Diamantina (MG). ana.marcia@ufv.br

Recebido para publicação em 11/05/2007 e aceito em 29/01/2008. 
influência significativa na qualidade da polpa. Esse parâmetro juntamente com o crescimento em volume e rendimento de polpa são as principais características para determinar os custos de produção da celulose (FONSECA et al., 1996). No entanto, a densidade básica é um parâmetro complexo que pode variar nas direções radial e longitudinal no tronco, entre árvores, entre espécies, com a idade, com o espaçamento e com o local de crescimento (DIAS e CLÁUDIO-DA-SILVA, 1991; ROCHA et al., 1995; CARNEIRO e AMARAL, 1997; DE ALENCAR et al., 2001).

Alguns estudos mostram que maiores rendimentos de polpação são alcançados se utilizadas madeiras com densidades variando entre 470-490 kg/m³ (DIAS e CLÁUDIO-DA-SILVA, 1991). Entretanto, os novos programas de melhoramento, visando à produção de polpa celulósica, são orientados para densidades de madeira com mais de $500 \mathrm{~kg} / \mathrm{m}^{3}$. Normalmente, o cozimento de madeira de alta densidade requer condições alcalinas mais fortes e temperaturas mais elevadas que penalizam o rendimento de polpa. Entretanto, a produtividade mais alta das madeiras com densidades maiores compensa em muito essa desvantagem. No total, madeiras com densidade mais alta mostram uma relação custo/benefício também mais alta no processamento para fabricação de celulose.

Em processos químicos de produção de polpa, a lignina é dissolvida, liberando as fibras (a fração holocelulósica). A presença de certos grupos funcionais como as metoxilas e estruturas condensadas de lignina influenciam a taxa de dissolução desta. Estudos com Eucalyptus grandis (PEREIRA et al., 1994) sugerem que, quanto mais alta a relação de siringila/guaiacila na lignina, mais baixa a quantidade de reagente consumida no processo de polpação. Os autores recomendam que essa taxa seja utilizada como critério na seleção de madeira para fabricação de polpa. Almeida e Silva (1997) avaliaram a influência dos extrativos da madeira na polpação e recomendou a inclusão do teor de extrativos como um critério de seleção de madeiras para produção de polpas Kraft, uma vez que, embora a maioria dos extrativos seja removida no início da polpação, eles consomem reagentes e afetam negativamente o produto final.

É importante avaliar o impacto da densidade básica e da composição química da madeira na economia total do processo, no desempenho da fábrica e na qualidade de polpa, particularmente em relação à produção de produtos diferenciados. O objetivo deste estudo foi avaliar impacto da densidade da madeira de Eucalyptus spp. e da sua composição química de carboidratos no rendimento de polpa, consumo específico da madeira, produtividade e qualidade da polpa de celulose produzida.

\section{MATERIAL E MÉTODOS}

Neste estudo, foram utilizados cavacos industriais de madeiras de dez espécies de eucalipto, com densidades básicas e composições químicas diferentes. Os cavacos foram coletados nos pátios de madeiras de empresas fabricantes de celulose brasileiras e chilenas (Eucalyptus globulus e Eucalyptus nitens). Por se tratar de cavacos industriais, não foi possível determinar com exatidão as procedências e as idades dos povoamentos de origem. Foram coletadas $20 \mathrm{~kg}$ de cavacos úmidos os quais foram selecionados de acordo com o método SCAN 40:01 (SCAN- test Methods, 2001). Os cavacos foram secados ao teor de umidade de aproximadamente 15\%. As madeiras foram codificadas como A (Eucalyptus globulus Labill.), B (Eucalyptus nitens (Deane \& Maiden) Maiden, C e D (o hibrido, Eucalyptus grandis W. Hill. Ex Maiden X Eucalyptus urophylla S.T. Blake), E, F, G e H (Eucalyptus grandis), I e J (Eucalyptus urophylla).

Os procedimentos da TAPPI (1998) foram utilizados para avaliar a madeira, a polpa e a qualidade do licor. As ligninas insolúvel e solúvel foram medidas utilizando os métodos de Gomide e Demuner (1986) e Goldschimid (1971) respectivamente. O teor de pentosanas foi medido segundo o método brometo/bromato (BROWNING, 1967). A relação siringila/guaiacila da lignina foi determinada como descrito por Lin e Dence (1992). Os carboidratos foram determinados por cromatografia a gás, conforme método-padrão da TAPPI (1998). Os grupos acetila foram determinados de acordo com o método descrito por Solar et al. (1987) e os ácidos hexenurônicos pelo método proposto por Vuorinen et al. (1996).

Os cozimentos kraft foram realizados em digestor rotativo, adaptado com quatro reatores de aço inox, de 2 litros cada um, tendo sido realizados oito cozimentos com kappa entre 15,5-16,7 para cada madeira. As condições usadas nos cozimentos foram: cavacos $=250 \mathrm{~g}$, temperatura máxima $=170^{\circ} \mathrm{C}$; sulfidez $=30 \%$; tempo até temperatura máxima $=90$ minutos; relação licor /madeira $=4 / 1$ e tempo à temperatura máxima = 90 minutos. Amostra de licor residual foi coletada para determinação do álcali ativo residual e teor de sólidos. 
O branqueamento foi feito com a seqüência OD(PO)D até alvura 90\% ISO. A deslignificação com oxigênio (O) e a extração oxidativa com peróxido (PO) foram realizado em reator/misturador pressurizado modelo Mark V, do Quantum Technologies Inc. Os estágios de dioxidação foram realizados em sacos de polietileno aquecidos em banho de vapor termostatizado. Amostra de licor residual foram coletadas após cada estágio de branqueamento para determinação de residuais. Todas as condições de branqueamento estão listadas na Tabela 1.

As polpas foram refinadas em um moinho PFI para 15 a $70^{\circ}$ SR (Grau Schopper Riegler). As propriedades de resistência da polpa foram avaliadas em função do grau Schopper Riegler em folhas de aproximadamente $60 \mathrm{~g} / \mathrm{m}^{2}$. Os testes de resistência à tração foram realizados em aparelho de testes INSTRON, modelo 4204, com sistema computadorizado de análise e aquisição de dados, com distância entre garras de $100 \mathrm{~mm}$, velocidade de teste de $25 \mathrm{~mm} / \mathrm{min}$ e capacidade da célula de carga de $1000 \mathrm{~N}$. Os testes de resistência ao rasgo, arrebentamento e passagem de ar foram realizados conforme métodos Tappi (TAPPI STANDARD METHODS, 1998), nos aparelhos Elmendorf, Mullen e Gurley respectivamente. As propriedades de capilaridade Klemm e índice de retenção de água foram determinadas conforme métodos Scan P13:64 (SCAN-test METHODS, 2001) e Tappi UM 256 (TAPPI STANDARD METHODS, 1998) respectivamente. As propriedades óticas foram medidas em aparelho Datacolor, modelo Elrepho 450X, e a mensuração das fibras em vídeo microscópio computadorizado e em aparelho de medição automática Galai CIS-100.

TABELA 1: Condições de branqueamento para a seqüência OD (PO) D.

TABLE 1: Bleaching Conditions for the OD(PO)D sequence.

\begin{tabular}{l|c|c|ccc}
\hline \multirow{2}{*}{ Condições } & \multirow{2}{*}{ Unidade } & \multicolumn{5}{|c}{ Estágios de Branqueamento ECF } \\
\cline { 3 - 6 } & & $\mathrm{O}$ & $\mathrm{Do}$ & $\mathrm{PO}$ & $\mathrm{D}$ \\
\hline Consistência & $\%$ & 10 & 10 & 10 & 10 \\
Oxigênio & $\mathrm{kPa}$ & 500 & - & 500 & - \\
Tempo de reação & $\mathrm{Min}$ & 60 & 60 & 90 & 180 \\
$\mathrm{pH}$ & - & 10,5 & 3 & 10,5 & 3,5 \\
Temperatura & ${ }^{\circ} \mathrm{C}$ & 100 & 80 & 80 & 70 \\
Dose de $\mathrm{O}_{2}$ & $\mathrm{~kg} / \mathrm{t}$ & 20 & - & 25 & - \\
Dose de $\mathrm{ClO}_{2}$ & $\mathrm{~kg} / \mathrm{t}$ & - & $\mathrm{KF} 2.4\left(^{* *}\right.$ & - & $\left(^{*}\right)$ \\
Dose de $\mathrm{H}_{2} \mathrm{O}_{2}$ & $\mathrm{~kg} / \mathrm{t}$ & - & - & 5 & - \\
Dose de $\mathrm{NaOH}_{\text {DaO }}$ & $\mathrm{kg} / \mathrm{t}$ & 20 & - & 12 & - \\
Dose de $\mathrm{H}_{2} \mathrm{SO}_{4}$ & $\%$ & - & $(*)$ & - & $\left(^{*}\right)$ \\
\hline
\end{tabular}

Em que: $(*)$ Carga de $\mathrm{ClO}_{2}$ para atingir $90 \%$ ISO de alvura e carga de $\mathrm{H}_{2} \mathrm{SO}_{4}$ para ajustar o $\mathrm{pH} ;\left(^{* *}\right) \mathrm{KF}=\left(\mathrm{kg} / \mathrm{t} \mathrm{ClO}_{2}\right.$ as $\mathrm{Cl}_{2}$ )/número kappa da polpa após o estágio $\mathrm{O}$.

\section{RESULTADOS E DISCUSSÃO}

\section{Caracterização da Madeira}

Os resultados das análises de fibras são apresentados na Tabela 2. Nenhuma variação significativa no comprimento de fibra foi observada em madeiras com diferentes densidades básicas. Entretanto, no geral com algumas exceções, madeiras menos densas apresentaram maiores diâmetros de fibra e de lúmen, menores teores de finos, menores espessuras de parede de fibra e menor valor de "coarseness". Fibras com menores espessuras de parede e maiores diâmetros de lúmen têm maior potencial de colapso, facilidade de refino, maior área de contato entre fibras na formação da folha de papel e, logo, maior resistência à tração. A resistência mecânica, juntamente com a opacidade, constitui-se no principal atributo de qualidade de papéis de escrita e impressão. Por outro lado, fibras de madeiras mais densas, com maior espessura de parede são mais rígidas e são indicadas para a fabricação de papéis absorventes de alto volume específico, alta capacidade de retenção de água e maciez e de menor lisura. Em geral, observaram-se correlações mais significativas das características morfológicas das fibras com a densidade básica que com a composição química de carboidratos da madeira. 
TABELA 2: Resultados da análise de fibras.

TABLE 2: Fiber analysis results.

\begin{tabular}{l|c|c|c|c|c|c|c|c|c|c}
\hline \multirow{2}{*}{ Resultados } & \multicolumn{10}{c}{ Polpas } \\
\cline { 2 - 12 } & $\mathrm{E}$ & $\mathrm{F}$ & $\mathrm{H}$ & $\mathrm{C}$ & $\mathrm{I}$ & $\mathrm{G}$ & $\mathrm{B}$ & $\mathrm{A}$ & $\mathrm{D}$ & $\mathrm{J}$ \\
\hline Densidade básica, $\mathrm{kg} / \mathrm{m}^{3}$ & 365 & 389 & 406 & 421 & 430 & 433 & 484 & 516 & 525 & 544 \\
Teor de carboidratos, \% & 70,5 & 73,1 & 74,5 & 70,0 & 72,1 & 70,9 & 70,3 & 72,6 & 70,4 & 70,7 \\
Comprimento de fibra, mm & 0,98 & 0,93 & 0,89 & 0,97 & 0,97 & 0,89 & 0,75 & 0,77 & 0,96 & 0,91 \\
Diâmetro de fibra, $\mu \mathrm{m}$ & 22,1 & 21,7 & 20,8 & 20,2 & 20,8 & 21,6 & 20,1 & 21,0 & 19,0 & 17,7 \\
Diâmetro de lúme, $\mu \mathrm{m}$ & 13,3 & 12,2 & 10,9 & 11,0 & 10,8 & 11,7 & 11,5 & 11,6 & 8,71 & 7,75 \\
Espessura de parede, $\mu \mathrm{m}$ & 4,39 & 4,73 & 4,98 & 4,62 & 5,02 & 4,97 & 4,32 & 4,68 & 5,17 & 4,99 \\
Número de fibras/gramas x 10 $\mathbf{1 0}^{6}$ & 33,8 & 28,1 & 32,2 & 31,5 & 25,5 & 28,5 & 38,0 & 25,4 & 26,1 & 25,1 \\
Coarseness, mg/100m de fibra & 4,58 & 5,65 & 4,93 & 5,21 & 6,09 & 5,57 & 4,99 & 6,67 & 6,20 & 6,52 \\
Teor de finos, \% & 8,56 & 8,56 & 6,34 & 8,05 & 8,56 & 7,27 & 7,84 & 7,87 & 7,27 & 7,65 \\
\hline
\end{tabular}

A Tabela 3 mostra que houve uma correlação mais alta entre a densidade básica e as características morfológicas das fibras do que entre a densidade básica e a composição química de carboidratos da madeira.

TABELA 3: Correlações entre densidade básica da madeira, composição química e características morfológicas.

TABLE 3: Correlations between wood basic density, chemical composition and morphological characteristics.

\begin{tabular}{l|cc}
\hline \multirow{2}{*}{ Variáveis } & \multicolumn{2}{|c}{ Carboidratos Totais } \\
\cline { 2 - 3 } & $\mathrm{R}$ & $\mathrm{r}$ \\
\hline Diâmetro de fibra & $-0,80^{*}$ & $0,34^{\mathrm{ns}}$ \\
Diâmetro de lúmen & $-0,76^{*}$ & $0,18^{\mathrm{ns}}$ \\
Espessura de parede & $0,32^{\mathrm{ns}}$ & $0,27^{\mathrm{ns}}$ \\
Número de fibras/grama & $-0,41^{\mathrm{ns}}$ & $-0,18^{\mathrm{ns}}$ \\
Coarseness & $0,75^{*}$ & $0,05^{\mathrm{ns}}$ \\
Teor de finos & $-0,27^{\mathrm{ns}}$ & $-0,29^{\mathrm{ns}}$ \\
\hline
\end{tabular}

Em que: * = Significativo em nível de $5 \%$ de probabilidade pelo teste $\mathrm{t}(\mathrm{P}<0,05)$; ns = Não-significativo em nível de $5 \%$ de probabilidade pelo teste $\mathrm{t}(\mathrm{P}>0,05)$.

A densidade básica e a composição química variaram consideravelmente entre as amostras de madeira como pode ser visto na Tabela 4. A madeira de Eucalyptus urophylla (amostra J) é a mais densa $\left(544 \mathrm{~kg} / \mathrm{m}^{3}\right)$ enquanto que a de Eucalyptus grandis (amostra E) é a mais leve $\left(365 \mathrm{~kg} / \mathrm{m}^{3}\right)$. As pentosanas variaram de 13,5\% (Eucaçyptus urophylla, amostra I) a 18,6\% (Eucalyptus globulus). Os extrativos solúveis de álcool/tolueno médios variaram de 1,28\% na amostra de Eucalyptus globulus (A) a 2,66\% na amostra de Eucalyptus urophylla (J). Em geral, os extrativos prejudicam o desempenham da polpação por causar incrustações nos equipamentos, encanamentos e tanques, formando "pitch" (ALMEIDA e SILVA, 1997). O teor de lignina diferiu em apenas 3,9\% entre as madeiras analisadas, com o menor teor (24,1\%) encontrado na amostra de Eucalyptus grandis $(\mathrm{H})$ e o maior (28,8\%) na amostra do hibrido Eucalyptus grandis $\mathrm{x}$ Eucalyptus urophylla (C). A remoção de lignina, que é o principal objetivo do processo de polpação, libera a porção de fibras da madeira e, portanto, um baixo teor de lignina e de extrativos na madeira favorece o desempenho da polpação e economizam reagentes. Os teores mais altos de hemiceluloses das amostras B, A, $\mathrm{J}$ e E sugerem que essas madeiras são mais indicadas para produtos de papel refinado. As hemiceluloses facilitam o refinamento e aumentam a ligação entre fibras, com conseqüente melhoria da resistência da folha.

A relação siringila/guaiacila da lignina variou entre as amostras de madeira, com um mínimo de 2,20 $\mathrm{mol} / \mathrm{mol}$ na amostra de Eucalyptus urophylla (J) e um máximo de $4,68 \mathrm{~mol} / \mathrm{mol}$ na amostra de Eucalyptus globulus (A). As estruturas de siringila da lignina são degradadas com mais facilidade durante a polpação, requerendo uma menor carga de álcali do que as estruturas guaiacila, assim resultando em menores perdas de rendimento (PEREIRA et al., 1994). De forma ideal, programas de melhoramento genético deveriam selecionar madeiras com maiores relações siringila/guaiacila, como as encontradas nas amostras A, B e G. O teor total de hemiceluloses da madeira foi calculado como sendo a soma de acetilas, xilanas, arabinanas, 
galactanas e glicomananas. Os ácidos 4-O-metilglucorônicos, também fazem parte da fração de hemiceluloses, mas estes não foram quantificados nesse estudo. Têm sido relatados valores de 3,1-4,6\% para esses ácidos em madeiras de Eucalyptus globulus e Eucalyptus nitens (WALLIS et al., 1996). A fração de glicose da glicomanana foi estimada partindo do teor de manose, com base nas relações manose:glicose relatadas na literatura. Valores na ordem de 1-2:1 têm sido relatados embora uma relação manose: glicose de 1:2,1 tenha sido encontrada para a madeira de Eucalyptus regnans (SJOSTROM, 1981). Nesse estudo, a relação manose:glicose foi assumida como sendo de 1:1. O teor de celulose foi calculado como sendo a glicose total menos a glicose na glicomanana. O teor de amido da madeira não foi levado em consideração nas análises de carboidratos. O teor de amido foi relatado previamente como sendo de 0,3-0,4\% em Eucalyptus globulus e de 1,0-1,4\% em Eucalyptus nitens (WALLIS et al., 1996).

A composição de carboidratos variou consideravelmente nas dez espécies de madeiras estudadas. $\mathrm{O}$ teor médio de celulose foi de 47,7\% com mínimo de 38,2\% e um máximo de 52,1\%, enquanto que o teor médio de hemiceluloses foi de 19,6\%, com um mínimo de 16,8\% e um máximo de 26,3\%. De forma ideal, um equilíbrio entre celulose e hemiceluloses deve ser buscado para que favoreça tanto o rendimento quanto as propriedades de refinamento da polpa.

TABELA 4: Densidade da madeira de Eucalyptus spp e sua composição química.

TABLE 4: Eucalyptus spp wood density and its chemical composition.

\begin{tabular}{|c|c|c|c|c|c|c|c|c|c|c|}
\hline \multirow[t]{2}{*}{ Análise Resultados } & \multicolumn{10}{|c|}{ Madeira } \\
\hline & $\mathrm{E}$ & $\mathrm{F}$ & $\mathrm{H}$ & $\mathrm{C}$ & $\mathrm{I}$ & $G$ & $\mathrm{~B}$ & A & $\mathrm{D}$ & $\mathrm{J}$ \\
\hline Densidade básica, $\mathrm{kg} / \mathrm{m}^{3}$ & 365 & 389 & 406 & 421 & 430 & 433 & 484 & 516 & 525 & 544 \\
\hline Teor de carboidratos, \% & 70,5 & 73,1 & 74,5 & 70,0 & 72,1 & 70,9 & 70,3 & 72,6 & 70,4 & 70,7 \\
\hline Lignina insolúvel, \% & 24,2 & 21,3 & 20,4 & 24,2 & 22,3 & 22,8 & 23,2 & 21,0 & 23,8 & 23,6 \\
\hline Lignina solúvel, \% & 3,7 & 3,8 & 3,7 & 3,8 & 3,3 & 4,0 & 4,6 & 5,1 & 3,8 & 3,0 \\
\hline Lignina total, \% & 27,9 & 25,1 & 24,1 & 28,0 & 25,6 & 26,8 & 27,8 & 26,1 & 27,6 & 26,6 \\
\hline $\begin{array}{l}\text { Extraíveis em etanol/ } \\
\text { tolueno, \% }\end{array}$ & 1,6 & 1,8 & 1,4 & 2,0 & 2,3 & 2,3 & 1,9 & 1,3 & 2,0 & 2,7 \\
\hline Matéria oxidável total, \% & 29,5 & 26,9 & 25,5 & 30,0 & 27,9 & 29,1 & 29,7 & 27,4 & 29,6 & 29,3 \\
\hline $\begin{array}{l}\text { Extraíveis em } \\
\text { diclorometano, \% }\end{array}$ & 0,32 & 0,28 & 0,07 & 0,18 & 0,22 & 0,29 & 0,29 & 0,28 & 0,17 & 0,26 \\
\hline Pentosanas, \% & 15,5 & 15,1 & 14,9 & 14,5 & 13,5 & 14,1 & 18,5 & 18,6 & 14,2 & 16,1 \\
\hline siringila/guaiacila & 2,73 & 2,87 & 2,90 & 2,47 & 2,56 & 3,20 & 3,32 & 4,68 & 2,62 & 2,20 \\
\hline Grupos acetila,\% & 2,8 & 2,6 & 2,9 & 2,7 & 2,6 & 2,6 & 3,6 & 3,3 & 2,5 & 2,7 \\
\hline Arabinanas, \% & 0,2 & 0,1 & 0,1 & 0,1 & 0,2 & 0,2 & 0,5 & 0,2 & 0,1 & 0,1 \\
\hline Xilanas, \% & 12,8 & 12,6 & 14,4 & 12,8 & 11,6 & 12,7 & 18,6 & 16,6 & 11,8 & 13,3 \\
\hline Mananas, \% & 1,1 & 0,7 & 0,9 & 1,0 & 0,9 & 0,5 & 1,4 & 1,2 & 0,8 & 1,0 \\
\hline Galactanas, \% & 0,9 & 1,1 & 0,5 & 0,9 & 0,8 & 1,3 & 0,8 & 1,3 & 0,8 & 1,0 \\
\hline Glicanas, \% & 49,7 & 50,0 & 49,1 & 51,8 & 53,0 & 49,3 & 39,6 & 43,6 & 49,0 & 51,0 \\
\hline Celulose, \% & 48,6 & 49,3 & 48,2 & 50,8 & 52,1 & 48,8 & 38,2 & 42,4 & 48,2 & 50,0 \\
\hline Hemiceluloses, \% & 18,9 & 17,8 & 19,7 & 18,5 & 17,0 & 17,8 & 26,3 & 23,8 & 16,8 & 19,1 \\
\hline Carboidratos, \% & 67,5 & 67,1 & 67,9 & 69,3 & 69,1 & 66,6 & 64,5 & 66,2 & 65,0 & 69,1 \\
\hline Acetila/10 unid. de xilose & 6,7 & 6,4 & 6,2 & 6,4 & 6,9 & 6,3 & 5,9 & 6,1 & 6,6 & 6,1 \\
\hline Metais: Ferro, ppm & 37,7 & 23,1 & 24,8 & 27,9 & 22,5 & 33,5 & 34,0 & 29,2 & 26,5 & 30,0 \\
\hline Manganês, ppm & 14,7 & 17,3 & 11,8 & 5,5 & 11,5 & 12,4 & 35,1 & 88,8 & 4,9 & 33,5 \\
\hline Cálcio, ppm & 457 & 517 & 578 & 390 & 423 & 400 & 533 & 1037 & 281 & 365 \\
\hline
\end{tabular}

O teor de grupos acetila está fortemente correlacionando com o teor de xilanas. De 5,9 a 6,9 grupos acetila foram detectados para cada dez unidades de xilose, com a madeira de Eucalyptus urophylla ( I ) apresentando a xilana menos acetilada, enquanto que a de Eucalyptus nitens apresenta a xilana mais acetilada. Esses valores concordam com os já relatados para Eucalyptus regnans no qual a xilana é cerca de 70\% acetilada (CARVALHO, 2002). Em geral, quanto maior o grau de acetilação, maior a perda de rendimento durante a polpação causada pelo aumento da demanda de álcali e perdas de peso da madeira em razão da desacetilação.

Nenhuma relação foi detectada entre o teor de metais e outras características da madeira. O 
manganês e o ferro são os principais metais que reagem com compostos oxigenados nos estágios de branqueamento, reduzindo a eficiência e aumentando o consumo de reagentes. As madeiras de Eucalyptus globulus (A) e de Eucalyptus urophylla (J) mostraram teores elevados de manganês. Para minimizar os impactos adversos dos metais, estágios ácidos ou quelantes podem ser incorporados no início da seqüência de branqueamento para solubilizar e remover ou inativar os metais. Existe também a opção de usar magnésio como estabilizador dos compostos oxigenados (COSTA e COLODETTE, 2002).

\section{Polpação Kraft}

Os resultados do cozimento kraft para o número Kappa 15,5-16,7 estão na Tabela 5. A densidade básica e a composição química de carboidratos da madeira exibiram fracas correlações com o rendimento e a carga de álcali ativo aplicada na polpação (Tabela 6).

Altos rendimentos de polpação (>53\%) e polpas de alta viscosidade foram obtidos para madeiras de densidade mais baixas (amostras E, H e I). Além disso, essas madeiras consumiram menos álcali ativo do que as madeiras de alta densidade e sofreram menos degradação de carboidratos e perda de viscosidade (Figura 1 e 2).

O consumo específico de madeira $\left(\mathrm{m}^{3}\right.$ por tonelada seca) foi calculado com base nas densidades e nos rendimentos de polpa. Consumo mais baixo foi obtido para madeira com alta densidade (B, A, D, J e G). Normalmente, a madeira é comercializada e transportada com base no volume. Portanto, quanto mais alta a densidade básica, mais celulose a madeira contém, e menores serão os custos variáveis da produção de polpa. Essa vantagem deve ser levada em consideração em programas de melhoramento florestal.

TABELA 5: Polpação Kraft de madeiras de Eucalyptus sp.

TABLE 5: Kraft pulping of Eucalyptus sp woods.

\begin{tabular}{|c|c|c|c|c|c|c|c|c|c|c|}
\hline \multirow[t]{2}{*}{ Análises/Resultados } & \multicolumn{10}{|c|}{ Polpas } \\
\hline & E & $\mathrm{F}$ & $\mathrm{H}$ & $\mathrm{C}$ & $\mathrm{I}$ & G & $\mathrm{B}$ & A & $\mathrm{D}$ & $\mathrm{J}$ \\
\hline Densidade básica, $\mathrm{kg} / \mathrm{m}^{3}$ & 365 & 389 & 406 & 421 & 430 & 433 & 484 & 516 & 525 & 544 \\
\hline Teor de carboidratos, \% & 70,5 & 73,1 & 74,5 & 70,0 & 72,1 & 70,9 & 70,3 & 72,6 & 70,4 & 70,7 \\
\hline Álcali ativo, \% & 15,3 & 14,8 & 15,7 & 16,8 & 15,7 & 15,4 & 18,5 & 16,0 & 16,3 & 16,9 \\
\hline Rendimento de polpa depurada, $\%^{(*)}$ & 53,2 & 55,6 & 53,3 & 51,0 & 53,5 & 52,8 & 48,9 & 52,6 & 52,0 & 51,6 \\
\hline Rejeitos, \% & 0,0 & 0,3 & 0,2 & 0,2 & 0,1 & 0,3 & 0,8 & 1,2 & 0,3 & 0,1 \\
\hline Rendimento total, \% & 53,2 & 55,9 & 53,5 & 51,2 & 53,6 & 53,1 & 49,7 & 53,8 & 52,3 & 51,7 \\
\hline Viscosidade, $\mathrm{cP}$ & 68,9 & 79,0 & 85,3 & 51,2 & 71,5 & 70,3 & 56,7 & 66,3 & 50,4 & 59,1 \\
\hline Alvura, \% ISO & 33,7 & 29,5 & 30,9 & 31,9 & 30,9 & 29,2 & 34,6 & 28,7 & 32,8 & 30,6 \\
\hline Ácido hexenurônico, mmol/kg & 46,1 & 41,7 & 45,0 & 44,0 & 41,1 & 39,9 & 46,2 & 36,9 & 41,1 & 49,6 \\
\hline Metais: Ferro, ppm & 25,2 & 28,1 & 24,8 & 27,8 & 21,6 & 29,3 & 104 & 50,2 & 69,2 & 27,4 \\
\hline Manganês, ppm & 4,3 & 5,4 & 2,9 & 1,7 & 3,6 & 4,4 & 13,2 & 82,2 & 3,2 & 10,5 \\
\hline Cálcio, ppm & 2665 & 2946 & 2703 & 1563 & 2334 & 2437 & 2266 & 3520 & 1965 & 2420 \\
\hline Álcali residual (AA), g/l & 3,5 & 3,9 & 3,9 & 5,4 & 4,8 & 4,4 & 6,0 & 5,0 & 5,1 & 5,3 \\
\hline pH do licor residual & 11,5 & 11,4 & 11,8 & 12,0 & 11,8 & 11,6 & 11,8 & 11,3 & 11,9 & 12,1 \\
\hline Teor de sólidos no licor, \% & 13,3 & 13,0 & 13,4 & 14,1 & 13,3 & 13,6 & 14,8 & 13,6 & 13,7 & 14,3 \\
\hline $\begin{array}{l}\text { Consumo específico de madeira, } \\
\mathrm{m}^{3} / \text { tpolpa depurada }\end{array}$ & 5,15 & 4,62 & 4,62 & 4,66 & 4,35 & 4,37 & 4,23 & 3,68 & 3,66 & 3,56 \\
\hline Sólidos totais no licor negro, $\mathrm{t} / \mathrm{t}$ & 1,28 & 1,17 & 1,28 & 1,40 & 1,27 & 1,29 & 1,55 & 1,31 & 1,35 & 1,38 \\
\hline
\end{tabular}

Em que: ${ }^{(*)}$ Desvio-padrão de oito cozimentos (tratamentos): 0,2 a 0,4.

Polpas não-branqueadas apresentaram cerca de 43,2 mmol/kg de ácidos hexenurônicos, variando de 39,9 a 49,6 mmol/kg. Essa variação foi significativa, considerando-se que condições constantes de polpação foram usadas, exceto pelo álcali ativo que variou na faixa de 14,8 a 18,5\%. Variações nos teores de ácidos hexenurônicos mostraram pouca relação com a carga de álcali utilizada no cozimento $\left(R^{2}=0,1845\right)$ e com o teor de pentosana na madeira $(\mathrm{R}=0,0037)$. Os valores de ácidos hexenurônicos relatados na literatura variam de 0,5 a $70 \mathrm{mmol} / \mathrm{kg}$ de polpa (WALLIS et al., 1996; COLODETTE et al., 2001; COSTA e COLODETTE, 2002; GOMIDE et al., 2000). Tipicamente as polpas Kraft de eucalipto têm valores de 40-60 mmol/kg (COLODETTE et al., 2001; COSTA e COLODETTE, 2002; GOMIDE et al., 2000).

Os altos níveis de ferro encontrados nas polpas oriundas das madeiras de Eucalyptus globulus (A), 
Eucalyptus nitens (B) e do hibrido Eucalyptus grandis x Eucalyptus urophylla (D) pode afetar negativamente o processo de branqueamento e a qualidade da polpa. Da mesma forma, o teor de manganês da polpa de Eucalyptus globulus (A) pode ter impacto negativo na deslignificação com oxigênio e no branqueamento. Concentrações de álcali ativo e o pH dos licores residuais são também apresentados na Tabela 5 . O pH final médio do licor negro residual foi relativamente baixo, atingindo 11,7, e valores de álcali residual menores que 5,0g NaOH/L. Menor quantidade de álcali foi consumido no cozimento das madeiras E, F, G e H, que produziram maiores rendimentos de polpa.

TABELA 6: Correlações de densidade básica de madeira e composição química de carboidratos com rendimentos de polpa depurada e carga de álcali ativo aplicado na polpação.

TABLE 6: Correlation of wood basic density and chemical composition with screened pulp yield and alkali charge used in pulping.

\begin{tabular}{l|cc|c|c|c|c}
\hline Variável & \multicolumn{2}{|c|}{ Densidade Básica } & \multicolumn{3}{c}{ Carboidratos Totais } \\
\cline { 2 - 8 } Dependente & Equação & $\mathrm{R}^{2}$ & $\mathrm{R}$ & Equação & $\mathrm{R}^{2}$ & $\mathrm{R}$ \\
\hline $\begin{array}{l}\text { Rendimento } \\
\text { depurado }\end{array}$ & $\mathrm{Y}=-0,0146 \mathrm{X}+59,05$ & 0,262 & $(-) 0,512$ & $\mathrm{Y}=0,7507 \mathrm{X}-1,2298$ & 0.4019 & 0.634 \\
Álcali ativo & $\mathrm{Y}=-0,0096 \mathrm{X}+11,817$ & 0,313 & 0,560 & $\mathrm{Y}=0,3597 \mathrm{X}+41,861$ & 0,2575 & $(-) 0,507$ \\
\hline
\end{tabular}

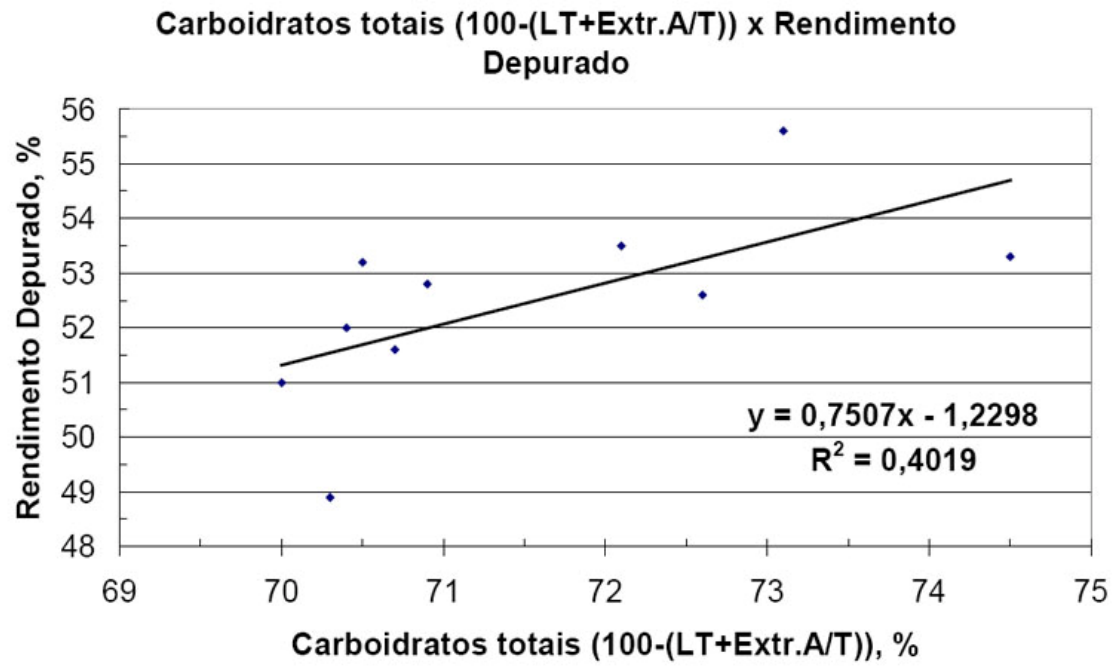

Em que: LT = Lignina Total; Extr.A/T = Extrativos em álcool tolueno.

FIGURA 1: Correlação entre teor carboidratos e rendimento depurado.

FIGURE 1: Correlation between total carbohydrates and screened yield. 


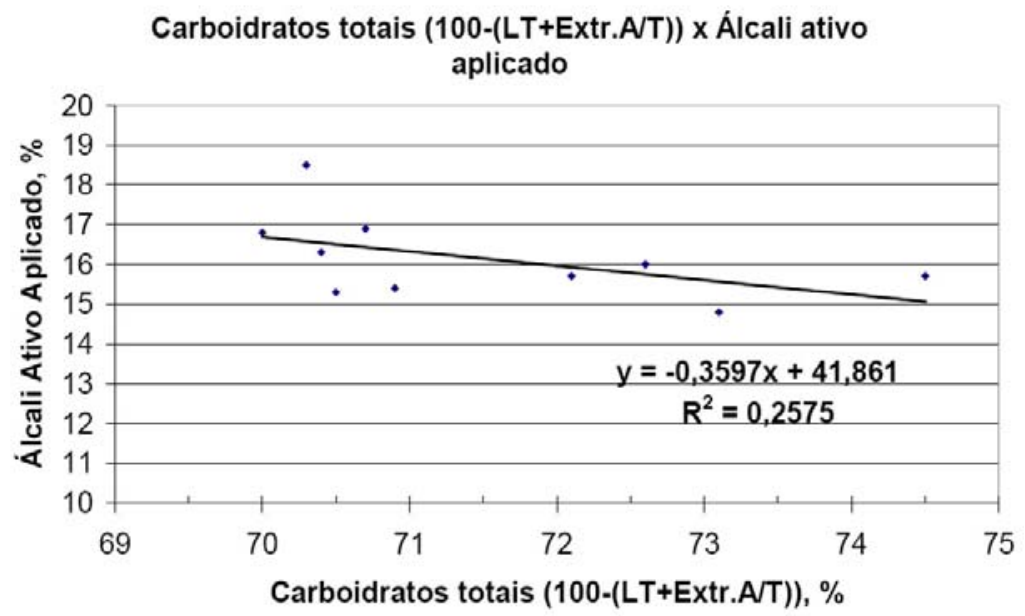

Em que: LT = Lignina Total; Extr.A/T = Extrativos em álcool tolueno.

FIGURA 2: Correlação entre carboidratos e álcali ativo empregado.

FIGURE 2: Correlation between total carbohydrate and active alkali applied.

\section{Deslignificação com oxigênio}

Após a deslignificação com oxigênio, o número kappa médio foi de 9,3, que é característico para indústrias de polpa Kraft de eucalipto (Tabela 7). A deslignificação com oxigênio foi mais seletiva (perda menor de viscosidade por unidade de número kappa) para polpas originadas de cozimento drástico ou de madeiras mais densas (A, D e J). Na deslignificação com oxigênio, não foi observada correlação entre a composição química de carboidratos da polpa e a taxa de deslignificação, ganho de alvura ou teor de carbono orgânico no licor residual.

\section{Branqueamento ECF}

Resultados de branqueamento são apresentados na Tabela 8. Os cálculos de rendimento total foram baseados em rendimento da polpação, deslignificação com oxigênio e de branqueamento. Embora uma perda média no rendimento de $2,0 \%$ tenha ocorrido no estágio de deslignificação e no branqueamento propriamente dito, essa perda não alterou a tendência dos resultados de rendimento estabelecidos após a polpação.

TABELA 7: Resultados após deslignificação a oxigênio.

TABLE 7: Results after oxygen delignification.

\begin{tabular}{l|c|c|c|c|c|c|c|c|c|c}
\hline \multirow{2}{*}{ Análises/Resultados } & \multicolumn{10}{c}{ Amostra de Madeira } \\
\cline { 2 - 12 } & $\mathrm{E}$ & $\mathrm{F}$ & $\mathrm{H}$ & $\mathrm{C}$ & $\mathrm{I}$ & $\mathrm{G}$ & $\mathrm{B}$ & $\mathrm{A}$ & $\mathrm{D}$ & $\mathrm{J}$ \\
\hline Densidade básica, kg/m & 365 & 389 & 406 & 421 & 430 & 433 & 484 & 516 & 525 & 544 \\
Teor de carboidratos, \% & 365,5 & 73,1 & 74,5 & 70,0 & 72,1 & 70,9 & 70,3 & 72,6 & 70,4 & 70,7 \\
N. kappa polpa não-branqueada & 15,5 & 15,7 & 16,5 & 16,7 & 16,4 & 16,1 & 15,7 & 15,7 & 16,1 & 16,7 \\
Número kappa polpa pré-O & 10,1 & 8,8 & 9,3 & 9,4 & 9,1 & 9,6 & 9,8 & 8,7 & 9,4 & 9,1 \\
Viscosidade polpa pré-O, mPa.s & 39,9 & 45,0 & 43,6 & 36,0 & 42,3 & 39,8 & 33,3 & 35,9 & 34,5 & 33,2 \\
Taxa de deslignificação, \% & 34,8 & 43,9 & 43,7 & 44,0 & 44,4 & 40,7 & 37,9 & 44,8 & 41,7 & 45,5 \\
Seletividade (viscosidade/n'kappa)* & 5,4 & 4,9 & 5,8 & 2,1 & 4,0 & 4,7 & 3,9 & 4,3 & 2,6 & 3,4 \\
Alvura, \% ISO & 49,1 & 49,0 & 50,7 & 51,3 & 50,7 & 47,8 & 48,6 & 43,3 & 48,4 & 49,6 \\
Ganho de Alvura na Pré-O, \% ISO & 15,4 & 19,5 & 19,8 & 19,4 & 19,8 & 18,6 & 14,0 & 14,6 & 15,6 & 19,0 \\
Rendimento, \% & 98,2 & 97,9 & 97,9 & 98,5 & 98,7 & 98,1 & 98,3 & 98,1 & 98,0 & 98,4 \\
TOC do filtrado pré-O, kg C/t polpa & 13,9 & 14,6 & 16,9 & 14,9 & 14,4 & 4,3 & 15,8 & 16,0 & 14,5 & 13,5 \\
\hline
\end{tabular}

Em que: $(*)$ Maior valor indica processo menos seletivo.

Em geral, com exceção da polpa de Eucalyptus globulus, as polpas consumiram 32-38 kg de Dioóxido de cloro $\left(\mathrm{ClO}_{2}\right)$, como cloro ativo, por tonelada de polpa. O desempenho da polpa de Eucalyptus globulus no branqueamento foi bem inferior ao das demais. Além do maior consumo $\mathrm{ClO}_{2} / \mathrm{t}$ para obtenção de 90\% ISO (48 $\mathrm{kg} \mathrm{ClO}_{2} / \mathrm{t}$ como cloro ativo), a alvura da polpa de Eucalyptus globulus foi sempre inferior nos 
estágios de branqueamento, e a reversão de alvura foi bem superior (2,8\% ISO) às das demais polpas (1,6$2,2 \%$ ISO). Esse fraco desempenho de alvura pode ser creditado aos elevados teores de metais, notadamente manganês e ferro, presentes na madeira e na polpa marrom derivada dessa espécie.

Filtrados residuais do branqueamento foram coletados após cada estágio de branqueamento e misturados para compor os efluentes do branqueamento. Esses efluentes não diferiram em termos de halogênios orgânicos absorvíveis (AOX), Demanda Química de Oxigênio (DQO), cor, Carbono Orgânico Total (COT) e pH. Nenhuma explicação lógica foi encontrada para o fato de que o halogênios orgânicos (OX) residual ser mais baixo em polpas de Eucalyptus globulus, uma vez que a carga de cloro ativo aplicada no branqueamento geralmente é indicativa de um teor mais alto de OX.

\section{Refinabilidade e propriedades de resistência de polpa}

Polpas de madeiras densas (A, B, D, J) mostraram comportamentos diferentes durante processo de refinamento (Figura 3). As polpas de Eucalyptus globulus (A) e Eucalyptus nitens (B) consumiram a menor energia para serem refinadas enquanto que as polpas de hibrido (D) e Eucalyptus urophylla (J) consumiram mais energia do que as outras. As propriedades de resistência são apresentadas para polpas não-refinadas e polpas refinadas a $30^{\circ} \mathrm{SR}$ (Tabela 9).

TABELA 8: Resultados do branqueamento da polpa com a seqüência D(PO)D, até alvura de 90\% ISO.

TABLE 8: Results of bleaching with the D(PO)D sequence to reach $90 \%$ ISO brightness.

\begin{tabular}{l|c|c|c|c|ccc|c|c|c}
\hline \multirow{2}{*}{ Variável } & \multicolumn{10}{c}{ Polpas } \\
\cline { 2 - 11 } & $\mathrm{E}$ & $\mathrm{F}$ & $\mathrm{H}$ & $\mathrm{C}$ & $\mathrm{I}$ & $\mathrm{G}$ & $\mathrm{B}$ & $\mathrm{A}$ & $\mathrm{D}$ & $\mathrm{J}$ \\
\hline Densidade básica, kg/m & 365 & 389 & 406 & 421 & 430 & 433 & 484 & 516 & 525 & 544 \\
Teor de carboidratos, \% & 70,5 & 73,1 & 74,5 & 70,0 & 72,1 & 70,9 & 70,3 & 72,6 & 70,4 & 70,7 \\
Número kappa após (PO) & 2,01 & 2,20 & 2,70 & 2,22 & 2,60 & 2,16 & 2,79 & 2,58 & 2,30 & 3,00 \\
Alvura após Do, \% ISO & 76,5 & 73,6 & 76,5 & 75,6 & 78,2 & 74,3 & 76,6 & 72,5 & 73,9 & 74,9 \\
Alvura após (PO), \% ISO & 86,8 & 87,6 & 87,8 & 86,5 & 88,8 & 85,9 & 85,6 & 78,7 & 87,2 & 86,9 \\
Alvura final, \% ISO & 90,0 & 90,0 & 90,1 & 90,1 & 90,1 & 90,0 & 90,0 & 90,0 & 90,2 & 90,2 \\
Reversão de Alvura, \% ISO & 1,7 & 1,5 & 2,0 & 1,6 & 1,8 & 2,4 & 1,6 & 2,8 & 2,1 & 2,2 \\
Número de cor posterior (NPC) & 0,15 & 0,10 & 0,25 & 0,15 & 0,30 & 0,35 & 0,25 & 0,15 & 0,15 & 0,25 \\
Viscosidade após (PO), m Pa.s & 19,6 & 24,2 & 25,8 & 18,2 & 22,9 & 18,7 & 21,5 & 18,9 & 20,4 & 21,8 \\
Viscosidade final, m Pa.s & 20,3 & 23,7 & 22,3 & 17,4 & 20,6 & 17,8 & 22,1 & 19,3 & 19,4 & 20,5 \\
ClO ${ }_{2}$ consumido, kg/t como Cl 2 & 35,7 & 32,1 & 33,6 & 33,9 & 33,6 & 37,9 & 35,4 & 47,8 & 35,1 & 36,8 \\
OX (polpa), g/t & 331 & 227 & 166 & 262 & 246 & 204 & 286 & 181 & 295 & 237 \\
AOX (efluente), kg/t & 0,45 & 0,53 & 0,50 & 0,41 & 0,48 & 0,47 & 0,50 & 0,57 & 0,46 & 0,50 \\
DQO (efluente), kg/t & 22,9 & 23,1 & 24,2 & 22,5 & 22,2 & 23,3 & 23,4 & 23,1 & 20,6 & 22,5 \\
COT (efluente), kg/t & 12,2 & 11,9 & 11,0 & 11,8 & 10,8 & 12,3 & 12,7 & 12,5 & 11,1 & 11,1 \\
Cor (efluente), kg Pt/t & 9,8 & 10,5 & 9,3 & 10,2 & 9,9 & 10,9 & 9,7 & 13,8 & 8,9 & 9,6 \\
Rendimento de branqueamento, \% & 96,7 & 96,6 & 96,9 & 97,0 & 96,7 & 96,7 & 96,7 & 97,1 & 97,3 & 96,3 \\
Rendimento total, \% & 50,5 & 52,6 & 50,6 & 48,7 & 51,1 & 50,1 & 46,5 & 50,1 & 49,6 & 48,9 \\
Consumo de madeira, m/tpolpa & 6,03 & 5,43 & 5,41 & 5,41 & 5,06 & 5,12 & 4,94 & 4,30 & 4,27 & 4,17 \\
branqueada & & & & & & & & &
\end{tabular}




\section{Graus Schopper- Riegler x Revoluções do Moinho PFI}

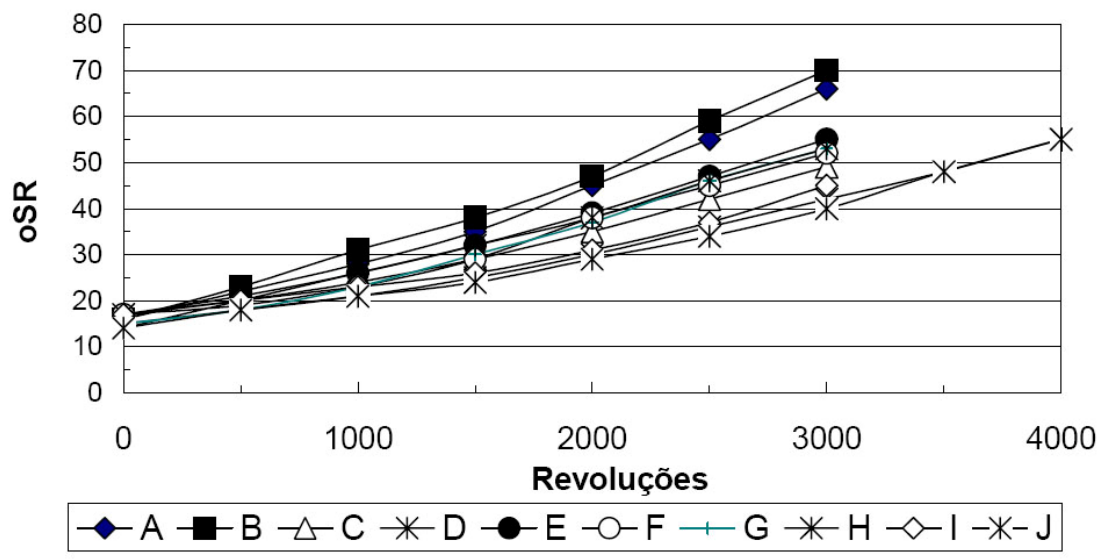

FIGURA 3: Curvas de refino para as dez amostras de polpa de madeira.

FIGURE 3: Refining curves for the ten wood pulp samples.

TABELA 9: Propriedades de resistências mecânicas e óticas de polpas não-refinadas (NR) e de polpas refinadas a $30^{\circ} \mathrm{SR}$.

TABLE 9: Strength and optical properties of unrefined (NR) pulps and pulps refined (R) to $30^{\circ} \mathrm{SR}$.

\begin{tabular}{|c|c|c|c|c|c|c|c|c|c|c|c|c|c|c|c|c|c|}
\hline \multirow[t]{2}{*}{ Polpas } & \multicolumn{2}{|c|}{$\begin{array}{l}\text { DEA } \\
\mathrm{kg} / \mathrm{m}^{3}\end{array}$} & \multicolumn{2}{|c|}{$\begin{array}{l}\text { VEA } \\
\mathrm{cm}^{3} / \mathrm{g}\end{array}$} & \multirow{2}{*}{$\begin{array}{c}\text { ER } \\
\text { Wh } \\
30^{\circ} \mathrm{SR} \\
\end{array}$} & \multicolumn{2}{|c|}{$\begin{array}{c}\text { IT } \\
\mathrm{Nm} / \mathrm{g}\end{array}$} & \multicolumn{2}{|c|}{$\begin{array}{c}\text { IR } \\
M N \cdot m^{2} / g\end{array}$} & \multicolumn{2}{|c|}{$\begin{array}{c}\mathrm{MOE} \\
\mathrm{MN} . \mathrm{m} / \mathrm{g}\end{array}$} & \multicolumn{2}{|c|}{$\begin{array}{l}\text { Lisura } \\
\text { S/50 cc }\end{array}$} & \multicolumn{2}{|c|}{$\begin{array}{c}\text { Opacidade } \\
\% \text { ISO }\end{array}$} & \multicolumn{2}{|c|}{$\begin{array}{l}\text { CEL } \\
\mathrm{kg} / \mathrm{m}^{2}\end{array}$} \\
\hline & NR & $\mathrm{R}$ & NR & $\mathrm{R}$ & & NR & $\mathrm{R}$ & NR & $\mathrm{R}$ & NR & $\mathrm{R}$ & NR & $\mathrm{R}$ & NR & $\mathrm{R}$ & NR & $\mathrm{R}$ \\
\hline $\mathrm{E}$ & 615 & 825 & 1,63 & 1,20 & 16,2 & 35,5 & 86,0 & 8,1 & 9,8 & 4,36 & 7,3 & 86,2 & 22,3 & 81,7 & 72,0 & 47,1 & 27,3 \\
\hline $\mathrm{F}$ & 546 & 760 & 1,84 & 1,32 & 20,0 & 27,1 & 66,0 & 7,2 & 10,5 & 3,90 & 6,8 & 86,1 & 21,7 & 76,6 & 69,0 & 43,9 & 29,3 \\
\hline $\mathrm{H}$ & 606 & 800 & 1,64 & 1,25 & 18,0 & 32,4 & 70,0 & 7,0 & 11,0 & 4,36 & 6,6 & 87,8 & 23,2 & 78,0 & 69,0 & 41,5 & 27,0 \\
\hline C & 533 & 775 & 1,88 & 1,27 & 20,0 & 30,0 & 80,0 & 6,0 & 10,3 & 4,05 & 6,8 & 86,8 & 22,3 & 80,7 & 71,5 & 46,0 & 26,8 \\
\hline I & 518 & 740 & 1,93 & 1,37 & 23,7 & 28,8 & 82,0 & 6,0 & 11,7 & 4,05 & 7,0 & 86,5 & 21,5 & 80,0 & 73,0 & 46,1 & 29,8 \\
\hline G & 521 & 740 & 1,92 & 1,35 & 21,0 & 31,1 & 78,0 & 5,5 & 10,5 & 4,34 & 7,1 & 87,1 & 22,3 & 78,9 & 72,0 & 43,8 & 28,3 \\
\hline B & 665 & 820 & 1,50 & 1,23 & 11,2 & 39,8 & 74,0 & 6,4 & 7,2 & 4,54 & 6,8 & 87,8 & 24,4 & 80,1 & 71,5 & 44,8 & 29,3 \\
\hline A & 525 & 740 & 1,90 & 1,35 & 15,0 & 31,2 & 69,0 & 4,7 & 8,5 & 4,17 & 6,5 & 86,3 & 21,5 & 77,7 & 71,0 & 42,6 & 28,0 \\
\hline $\mathrm{D}$ & 430 & 710 & 2,33 & 1,39 & 27,5 & 22,4 & 74,0 & 3,4 & 10,3 & 3,55 & 6,9 & 87,8 & 23,3 & 78,9 & 70,3 & 42,3 & 28,5 \\
\hline $\mathrm{J}$ & 461 & 675 & 2,18 & 1,50 & 26,2 & 26,7 & 64,0 & 4,6 & 10.7 & 4,07 & 6,4 & 87,6 & 27,5 & 78,8 & 73,5 & 43,6 & 31,8 \\
\hline
\end{tabular}

Em que: $\mathrm{ER}=$ energia de refino; $\mathrm{NR}$ = não-refinada, $\mathrm{R}=$ refinada $\left(\mathrm{a} 30^{\circ} \mathrm{SR}\right), \mathrm{DEA}=$ densidade específica aparente, VEA = volume específico aparente, $\mathrm{IT}=$ índice de tração, IR = índice de rasgo, $\mathrm{MOE}=$ módulo de elasticidade, $\mathrm{CEL}=$ coeficiente de dispersão de luz.

A combinação de gramatura e espessura, que são duas propriedades importantes em papel, conduz à densidade específica aparente (DEA) e volume específico aparente (VEA). Polpas não-refinadas (madeiras A, D e J) produziram papéis menos densos (volume específico mais alto), que são apropriados para o segmento de papéis sanitários. Em geral, houve uma relação melhor da densidade básica com as propriedades de resistência e óticas do que dessas propriedades com a composição química de carboidratos das madeiras (Tabela 10).

Embora a literatura científica (FONSECA et al., 1996, CARNEIRO e AMARAL, 1997) sugira que madeiras de menor densidade resultam em polpas de melhores resistências à tração, essa tendência não foi observada neste estudo (Tabela 9). Não houve boa correlação entre densidade básica da madeira e as propriedades de tração, rasgo e opacidade da polpa. Alta resistência à tração e boa opacidade são necessários para fabricação de papéis de impressão e escrita, embora a melhoria de uma dessas duas propriedades implica, geralmente, em piora da outra. Carpim et al. (1987) afirmaram que a estrutura anatômica da madeira é o fator determinante das propriedades óticas e de resistência da polpa. Fibras de paredes espessas e rígidas formam papéis com alto volume específico, com um número maior de interfaces, que refletem a luz incidente resultando numa opacidade mais alta. Além disso, o número de fibras por grama da polpa determina o número de interfaces ar-fibra e, conseqüentemente, o coeficiente de dispersão de luz (CDL) e a opacidade. 
TABELA 10: Correlação da densidade básica e da composição química de carboidratos das madeiras com as propriedades de resistência da polpa refinada a $30^{\circ} \mathrm{SR}$.

TABLE 10: Correlation of basic density and wood carbohydrate chemical composition with strength properties of pulp refined to $30^{\circ} \mathrm{SR}$.

\begin{tabular}{|c|c|c|c|c|c|c|}
\hline \multirow{2}{*}{$\begin{array}{l}\text { Variável } \\
\text { dependente }\end{array}$} & \multicolumn{3}{|c|}{ Densidade básica $\left(\mathrm{Kg} / \mathrm{m}^{3}\right)$} & \multicolumn{3}{|c|}{ Carboidratos totais (\%) } \\
\hline & Equação & $\mathrm{R}^{2}$ & $\mathrm{R}$ & Equação & $\mathrm{R}^{2}$ & $\mathrm{R}$ \\
\hline $\begin{array}{l}\text { Energia de refino } \\
\text { (Wh) }\end{array}$ & $Y=0,0244 X+8,8804$ & 0,089 & 0,30 & $Y=-0,4353 X+51,006$ & 0,017 & $(-) 0,13$ \\
\hline $\begin{array}{l}\text { Î́ndice de tração } \\
(\mathrm{Nm} / \mathrm{g})\end{array}$ & $Y=-0,0591 X+100,98$ & 0,259 & (-) 0,51 & $Y=-2,0435 X+220,43$ & 0,180 & (-) 0,42 \\
\hline $\begin{array}{l}\text { Índice de rasgo } \\
\left(\mathrm{MN} \cdot \mathrm{m}^{2} / \mathrm{g}\right)\end{array}$ & $Y=-0,0055 X+12,541$ & 0,065 & (-) 0,26 & $Y=0,2417 X-7,2075$ & 0,075 & 0,27 \\
\hline Módulo de & & & & & & \\
\hline $\begin{array}{l}\text { Elasticidade } \\
\text { (MN.m/g) }\end{array}$ & $Y=-0,0027 X+8,0407$ & 0,372 & (-) 0,61 & $Y=-0,064 X+11,397$ & 0,121 & $(-) 0,35$ \\
\hline Opacidade (\%ISO) & $Y=0,0086 X+67,447$ & 0,111 & 0,33 & $Y=-0,6297 X+116,36$ & 0,347 & $(-) 0,59$ \\
\hline Lisura (S/50cc) & $Y=0,0184 X+14,742$ & 0,335 & 0,58 & $Y=-0,3932 X+51,166$ & 0,089 & (-) 0,30 \\
\hline $\begin{array}{l}\text { Reflectância } \\
\left(\mathrm{Kg} / \mathrm{m}^{2}\right)\end{array}$ & $Y=0,0136 X+22,492$ & 0,290 & 0,54 & $Y=-0,1444 X+38,959$ & 0,019 & (-) 0,14 \\
\hline
\end{tabular}

As propriedades de superfície dos papéis mais comumente avaliadas são lisura e rugosidade. Ambas podem indicar irregularidades na superfície do papel durante a consolidação da folha, as quais afetam o uso final do produto. A suavidade está associada à ausência de maciez e é analisada pelo toque. Medidas de lisura (Tabela 9) mostraram que madeiras densas (A, D e J) com fibras rígidas produzem papéis mais ásperos. Uma comparação de propriedades higroscópicas e de permeabilidade do papel é apresentada na Tabela 11. Papéis produzidos das várias amostras, partindo de polpas não-refinadas, não apresentaram diferenças significativas na resistência à passagem de ar entre amostras. Entretanto, polpas refinadas, originadas de madeiras com mais fibras por grama, formaram papéis compactos que são mais resistentes à passagem de ar, como foi confirmado para Eucalyptus nitens (amostra B), do hibrido Eucalyptus grandis $x$ Eucalyptus urophylla (amostra C) e Eucalyptus urophylla (amostra J); mas não se expressou na polpa de Eucalyptus grandis (amostra E), que possuía 33,78 x $10^{6}$ fibras/grama.

TABELA 11: Permeabilidade e propriedades higroscópicas de polpas não refinadas e refinadas a (30 SR). TABLE 11: Permeability and hygroscopic properties of unrefined pulps and pulps refined to $30^{\circ} \mathrm{SR}$.

\begin{tabular}{|c|c|c|c|c|c|c|c|c|c|c|c|}
\hline \multirow[t]{2}{*}{ Polpas } & \multicolumn{2}{|c|}{$\begin{array}{l}\text { DEA } \\
\mathrm{kg} / \mathrm{m}^{3}\end{array}$} & \multicolumn{2}{|c|}{$\begin{array}{l}\text { VEA } \\
\mathrm{cm}^{3} / \mathrm{g}\end{array}$} & $\begin{array}{l}\text { ER } \\
\text { Wh }\end{array}$ & \multicolumn{2}{|c|}{$\begin{array}{c}\text { RPA } \\
\text { s/100cc }\end{array}$} & \multicolumn{2}{|c|}{$\begin{array}{l}\text { WRV } \\
\%\end{array}$} & \multicolumn{2}{|c|}{$\begin{array}{c}\text { Capilaridade Klemm } \\
\text { cm }\end{array}$} \\
\hline & NR & $\mathrm{R}$ & NR & $\mathrm{R}$ & $30^{\circ} \mathrm{SR}$ & NR & $\mathrm{R}$ & NR & $\mathrm{R}$ & NR & $\mathrm{R}$ \\
\hline $\mathrm{E}$ & 615 & 825 & 1,63 & 1,20 & 16,2 & 0,7 & 13,0 & 172,4 & --- & 9,5 & 4,5 \\
\hline $\mathrm{F}$ & 546 & 760 & 1,84 & 1,32 & 20,0 & 4,2 & 42,0 & 197,2 & --- & 5,7 & 2,7 \\
\hline $\mathrm{H}$ & 606 & 800 & 1,64 & 1,25 & 18,0 & 1,4 & 30,0 & 177,6 & --- & 7,8 & 3,0 \\
\hline C & 533 & 775 & 1,88 & 1,27 & 20,0 & 0,4 & 13,0 & 153,3 & --- & 11,7 & 3,5 \\
\hline I & 518 & 740 & 1,93 & 1,37 & 23,7 & 0,2 & 0,2 & 211,1 & --- & 6,1 & 2,7 \\
\hline G & 521 & 740 & 1,92 & 1,35 & 21,0 & 1,0 & 18,0 & 196,7 & --- & 8,9 & 3,5 \\
\hline B & 665 & 820 & 1,50 & 1,23 & 11,2 & 1,0 & 22,0 & 187,9 & --- & 9,5 & 3,7 \\
\hline A & 525 & 740 & 1,90 & 1,35 & 15,0 & 1,6 & 34,0 & 209,6 & --- & 7,4 & 2,7 \\
\hline D & 430 & 710 & 2,33 & 1,39 & 27,5 & 1,1 & 22,0 & 196,9 & --- & 8,6 & 3,2 \\
\hline $\mathrm{J}$ & 461 & 675 & 2,18 & 1,50 & 26,2 & 0,4 & 8,0 & 172,8 & --- & 12,1 & 4,5 \\
\hline
\end{tabular}

Em que: $\mathrm{ER}$ = energia de refino; $\mathrm{NR}$ = não-refinada, $\mathrm{R}$ = refinada (a 30 $\left.{ }^{\circ} \mathrm{SR}\right), \mathrm{DEA}=$ densidade específica aparente, VEA = volume específico aparente, RPA = resistência à passagem de ar, WRV = Índice de retenção de água.

Produtos de papel não-refinado ou ligeiramente refinado incluem toalhas de papel, guardanapos, papéis faciais, fraldas etc. As características mais importantes de qualidade associadas a esses produtos são suavidade (propriedade de superfície) e absorção e retenção de líquidos. Na sua fabricação, são usadas matérias-primas que resultam em papéis de baixa densidade e alto volume específico, com potencial para 
absorver e reter grande quantidade de líquido. Carpin et al. (1987) e Dias e Cláudio-da-Silva (1991) afirmaram que fibras mais rígidas, com paredes espessas favorecem a produção de papéis com volume específicos mais altos. A experiência industrial em fábrica de celulose kraft branqueada de eucalipto tem mostrado que uma polpação mais drástica, com produção de polpa marrom de número kappa comparativamente mais baixo, proporciona polpas com baixo teor de hemiceluloses e, dessa forma, com estrutura mais aberta e menor densidade de ligações químicas inter e intra-fibrilares, favorecendo a retenção de água. Em geral, dois testes são realizados para medir esse atributo do papel, o Índice de Retenção de Água (WRV - Water Retention Value) e a Capilaridade Klemm. Os resultados na Tabela 11 mostraram que polpas produzidas com madeiras de baixa densidade (E, H e I) apresentaram índices de retenção de água mais altos do que polpas feitas de madeiras com densidade alta. Com o refino, a estrutura do papel torna-se mais consolidada, reduzindo o diâmetro médio aparente dos capilares no papel. Conseqüentemente, a mobilidade ascendente do filme de água é reduzida, diminuindo a capilaridade Klemm (Levlin e Söderhjelm, 2000). O refino teve menos impacto em polpas oriundas de madeiras mais densas com fibras rígidas que em polpas derivadas de madeiras mais leves.

\section{CONCLUSÕES}

Os resultados permitiram concluir que a densidade básica de madeiras de eucalipto correlaciona-se mais fortemente com as características morfológicas das fibras e qualidade da polpa do que a sua composição química. Porém o rendimento da polpação é mais influenciado pela composição química da madeira. Madeiras de eucalipto menos densas produziram maiores rendimentos de polpação, porém maior consumo específico de madeira. Sugere-se que as madeiras menos densas de eucalipto sejam as mais adequadas para a produção de papéis de impressão e de escrita, enquanto que as madeiras mais densas sejam mais adequadas para o segmento de papéis sanitários.

\section{REFERÊNCIAS BIBLIOGRÁFICAS}

ALMEIDA, J. M.; SILVA. D. J. Inclusão de um novo e importante parâmetro potencial de seleção de eucalipto para produção de polpa kraft. IUFRO, v. 3, p. 69, 1997.

BROWNING, B. L. Methods of wood chemistry. New York : Wiley-Interscience, 1967, p.387-414. v.2

CARNEIRO, C. J. G.; AMARAL, C. A. S. S. Caracterização da variabilidade longitudinal da árvore visando a produção de celulose. In: CONGRESSO ANUAL DA ABTCP, 30.,1997, São Paulo. Anais... São Paulo, 1997. p. 271. CARPIM, M. A. et al. A influência do núimero de fibras por grama nas propriedades oticas do papel. In: CONGRESSO ANUAL DA ABTCP, 20.,1987, São Paulo. Anais... São Paulo, 1987. p. 183.

CARVALHO, A. G. M. Relação siringila: guaiacila na lignina e suas implicações. 2002. 40 f. Monografia (Pósgraduação lato sensu em Tecnologia de Celulose e Papel) - Universidade Federal de Viçosa, Viçosa.

COLODETTE, J. L. et al. Yield and bleachability of hardwood and softwood kraft polysulphide pulps. Pulp \& Paper Canada, v. 102, n. 9, p. 269-272, 2001.

COSTA, M. M.; COLODETTE, J. L. Efeito da composiçao química da polpa kraft- $\mathrm{O}_{2}$ na sua branqueabilidade. O Papel, v. 8, p. 93-103, 2002.

DE ALENCAR, G. S. B.; BARRICHELLO, L. E. G.; SILVA, JR., F. G. Qualidade da madeira de híbrido de E. grandis x E. urophylla e seleção precoce. In: CONGRESSO ANUAL DA ABTCP, 34., 2001, São Paulo. Anais... São Paulo, 2001.

DIAS, V. R. L.; CLÁUDIO-DA-SILVA, JR. E. Pulp and paper properties as influence by wood density - same species and age of Eucalyptus. In: INTERNATIONAL PAPER PHYSICS CONFERENCE, 1., 1991, Kailua Kona, Hawai. Proceedings.... Kailua Kona, Hawai, 1991.

FONSECA, S. M.; OLIVEIRA, R. C.; SILVEIRA, P. N. Seleção da árvore industrial : procedimentos, riscos, custos e beneficios. Revista Árvore, v. 20, n. l, p. 69-85, 1996.

GOLDSCHIMID, O. Ultraviolet spectra. In: SARKANEN, K. V.; LUDWIG, C. H. Lignins: occurrence, formation, structure and reactions. New York : John Wiley \& Sons, 1971. p. 241-266.

GOMIDE, J. L. et al. Fatores que afetam a branqueabilidade de polpas kraft de Eucalyptus 2: influência de parâmetros da polpação. O Papel, n. 12, p. 61-70, 2000.

GOMIDE, J. L.; DEMUNER, B. J. Determinação do teor de lignina em material lenhoso: Método Klason modificado. O Papel, v. 47, n. 8, p. 36-38, 1986.

LEVLIN, J-E.; SODERHJELM, L. Pulp and paper testing. In: GULliCHSEN, J.; PAULAPURO, H. (eds.). 
Papermaking science and technology. Atlanta-USA: Tappi Press, 2000. v.17

LIN, S. Y.; DENCE, C. W. Methods in Lignin Chemistry. Berlin : Springer-Verlag, 1992. 578 p.

PEREIRA, D. E. D. et al. A relação guaiacila/siringila como critério de seleção de árvores e sua importância no processo de deslignificação. In: CONGRESSO LATINO-AMERICANO DE DESLIGNIFICAÇÃO, 1., 1991, Vitória. Anais... Vitória: ABTCP, 1994, p.21-31.

ROCHA, M. G. B. et al. Estratégia para melhoramento das características teenológicas de Eucalyptus sp. na Cenibra. In: WORKSHOP SOBRE QUALIDADE DO PRODUTO FINAL, 1995, Belo Horizonte. Anais... Belo Horizonte: SIF, 1995.

SCAN-test METHODS Scandinavian Pulp, Paper and Board Testing Committée, Stockholm, Sweden. 2001.4 p.

SHIMOYAMA, V. R. S.; BARRICHELLO, L. E. G. Influência de características anatômicas e químicas sobre a densidade básica dc madeira de Eucalyptus sp. In: CONGRESSO ANUAL DA ABTCP, 24., 1991, São Paulo. Anais... São Paulo: ABTCP. 1991. p. 23-36.

SJÖSTROM, E. Wood chemistry, fundamentals and applications. New York : Academic Press, 1981. p.293.

SOLAR, R.; KACIK, F.; MELCER, I. Simple semimicro method for the determination of o-acetyl group in wood and related materials. Nordic Pulp \& Paper Research Journal, v. 4, p. 139-141, 1987.

TAPPI TECHNICAL DIVISIONS AND COMMITTEES. TAPPI Test Methods, Atlanta, 1998.

VUORINEN, T. et al. Selective hydrolysis of hexenuronic acid groups and its application in ECF and TCF bleaching of kraft pulps. In: INTERNATIONAL PULP BLEACHING CONFERENCE, 1996, Washington. Proceedings... Washington, USA, 1996. p. 43-51.

WALLIS, A. F. A.; WEARNE, R. H.; WRIGHT, P. J. Chemical analysis of polysaccharides in plantation eucalypt woods and pulps. Appita Journal, v. 49, n. 4, p. 258-262, 1996. 